\title{
Micellar Nanoparticle-encapsulated Epirubicin
}

National Cancer Institute

\section{Source}

National Cancer Institute. Micellar Nanoparticle-encapsulated Epirubicin. NCI Thesaurus.

Code C139003.

A nanoparticle-based prodrug formulation consisting of polymeric micelles encapsulating the anthracycline epirubicin, with potential antineoplastic activity. Epirubicin is covalently bound to polyethylene glycol (PEG) polyaspartate block copolymers through an acid-labile hydrazone bond and, upon suspension in an aqueous solution, a micellar structure with an outer hydrophilic PEG shell surrounding the hydrophobic epirubicin is formed. Upon administration of the micellar nanoparticle-encapsulated epirubicin, the nanoparticles are stable in the bloodstream and specifically accumulate in the tumor tissue. Due to the acidic conditions in the tumor and the $\mathrm{pH}$-responsive nature of the micelles, epirubicin is released in the tumor milieu; it then intercalates into DNA and inhibits topoisomerase II, which inhibits DNA replication and interferes with synthesis of both RNA and protein. Compared to the administration of epirubicin alone, this formulation increases the watersolubility of epirubicin and increases its therapeutic effect while decreasing its cardiotoxicity. 